\title{
Synthesis and Characterization of Branched Metallic Nanostructures
}

\author{
Justin L. Burt, Jose Luis Elechiguerra, Jose Reyes-Gasga, Miguel Jose-Yacaman
}

Department of Chemical Engineering, Texas Materials Institute, Center for Nano- and Molecular Science and Technology, The University of Texas at Austin, Austin, Texas 78712-1062

Metallic nanostructures exhibit unique properties and are of growing interest in a wide range of applications including catalysis, electronics, optics, and biosensing [1-4]. The size and shape of nanocrystals determine their physicochemical properties, so control over their morphology is critical. The self-assembly of nanocrystals into superlattices has been the subject of intense research, and nanostructures with specific shapes could be used to direct the growth of such assemblies [5].

Synthesis of metallic nanostructures via chemical reduction typically yields regular crystals with aspect ratios $\sim 1$, so strategies for the formation of nanocrystals with more exotic shapes (i.e. rods and wires) have been developed. Branched nanostructures are also highly desirable. Great progress has been made in the synthesis of branched semiconductor nanocrystals [6]. Recently, the synthesis of the first branched gold nanostructures has been reported [7-8]. Polytypism exists for open, tetrahedrally bonded structures such as semiconductors, but branched gold nanocrystals have FCC structure, so polytypism is not involved. To date, the growth mechanism for branched gold nanostructures remains elusive.

The synthesis of branched gold nanostructures evolved from the seed-mediated growth techniques developed for gold nanorods. As a result, many variables previously studied in gold nanorod synthesis have been identified as critical for the growth of branched gold nanostructures, including the ratio of seed crystals to ionic precursor, the concentration of surfactant, and the addition of silver ions to the growth medium.

We have discovered that branched gold nanostructures can also be obtained without the use of seed particles or surfactants. Our synthesis is carried out in aqueous solution at ambient conditions, and the only starting materials used are chloroauric acid and ascorbic acid. These branched gold nanostructures are a direct result of the interaction between gold and ascorbic acid. TEM analysis was performed using a JEOL $2010 \mathrm{~F}$ microscope equipped with Schottky-type field emission gun, ultra-high resolution pole piece $(\mathrm{Cs}=0.5 \mathrm{~mm})$, and a STEM unit with high angle annular dark field (HAADF) detector operating at $200 \mathrm{kV}$. A double tilt sample holder was used to perform a 3D analysis of the branched nanoparticle morphology. In the course of our analysis, we employed techniques including weak-beam dark-field microscopy and HAADF.

\section{References:}

[1] C.B. Murray et al., Annu. Rev. Mater. Sci. (2000), 30, 545-610.

[2] M. José-Yacamán et al., J. Vac. Sci. Tehcnol. B (2001), 19, 1091-1103.

[3] H. Bönnemann, R.M. Richards, Eur. J. Inorg. Chem. (2001), 2455-2480.

[4] J.-M. Nam et al., Science (2003), 301, 1884-1886.

[5] H. Liu, A.P. Alivisatos, Nano Lett. (2004), 4, 2397-2401.

[6] L. Manna et al., J. Am. Chem. Soc. (2000), 122, 12700-12706.

[7] S. Chen et al., J. Am. Chem. Soc. (2003), 125, 16186-16187.

[8] T.K. Sau, C.J. Murphy, J. Am. Chem. Soc. (2004), 126, 8648-8649. 

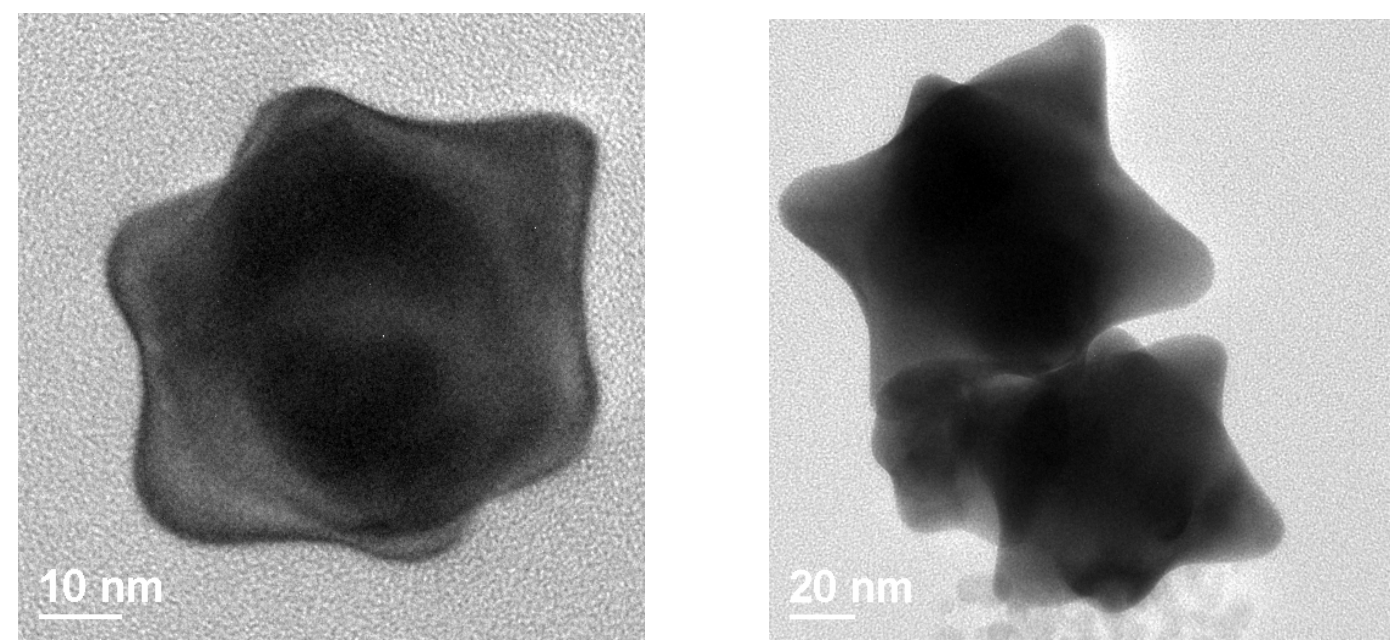

Figure 1. Branched gold nanostructures, obtained without seed crystals or surfactant molecules.
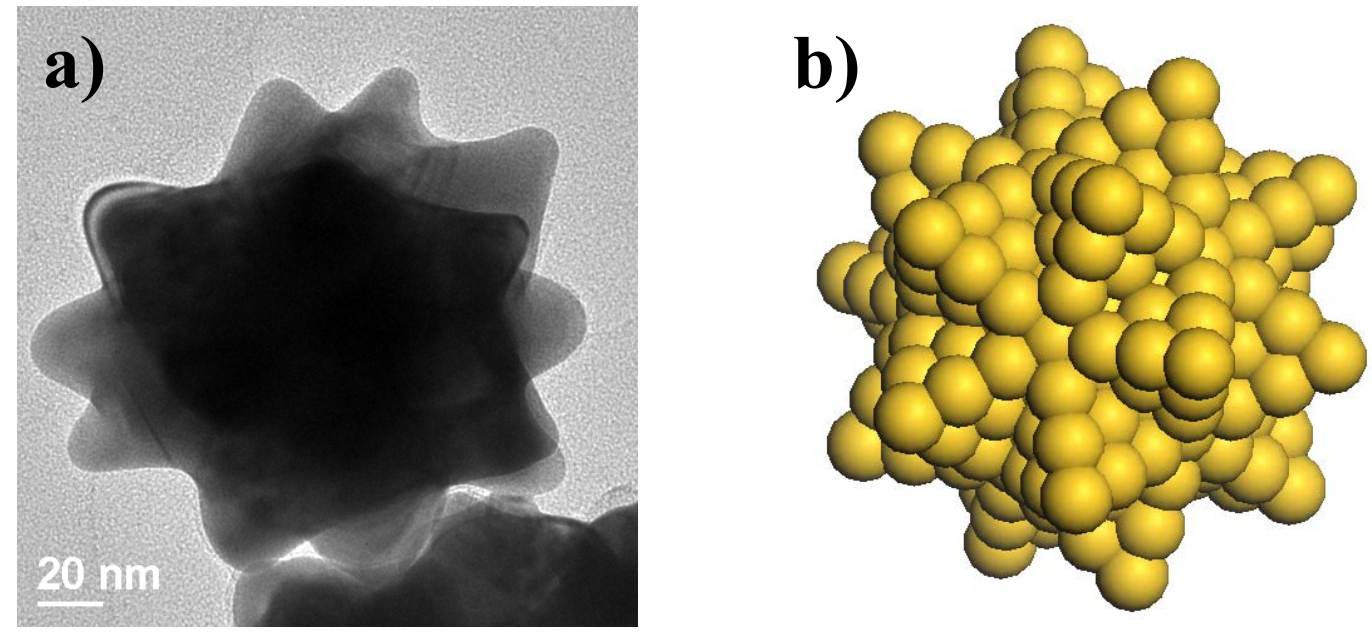

Figure 2. Multibranched gold nanoparticle, a) experimental image and b) proposed model. The nanoparticle was tilted to obtain 3D reconstruction, which corresponds to the proposed morphology.
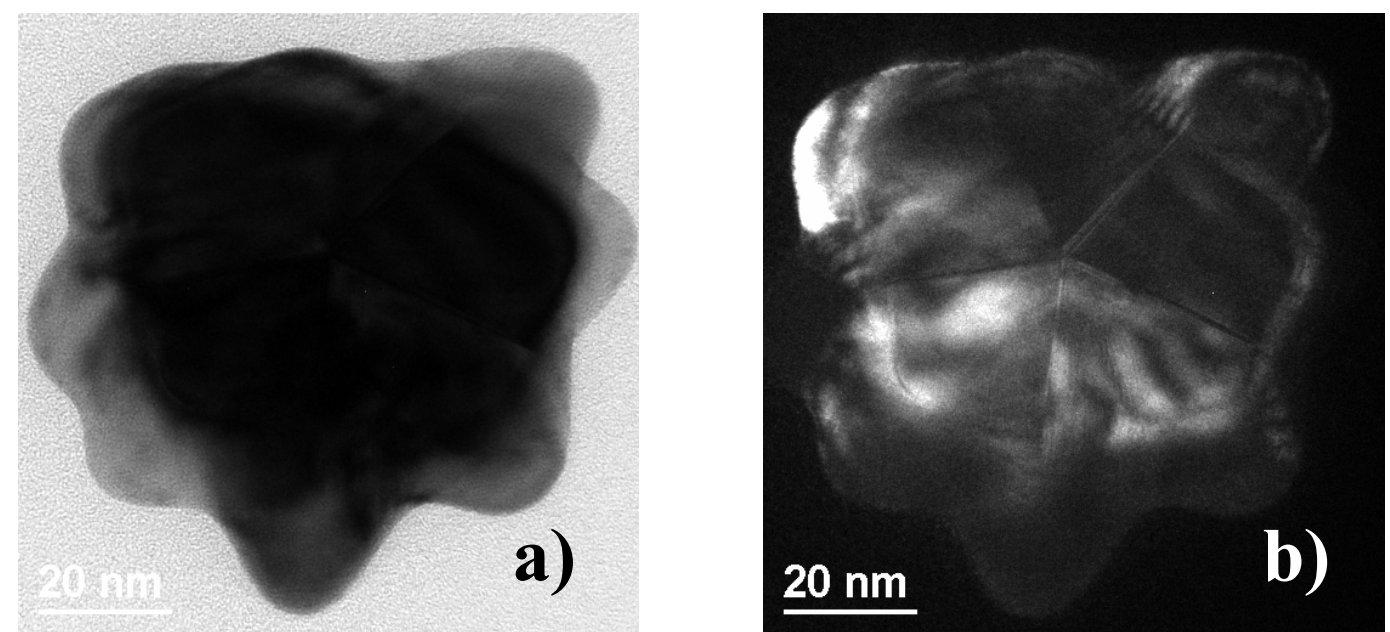

Figure 3. Branched gold nanoparticle imaged with a) bright field and b) weak-beam dark-field. The dark field image clearly reveals the five-fold symmetry perpendicular to the beam axis. 